\title{
Pluto images clinch the existence of water, methane, and life on this typical dark matter planet (Withdrawal Notice)
}

\section{Carl Gibson}

Carl H. Gibson, "Pluto images clinch the existence of water, methane, and life on this typical dark matter planet (Withdrawal Notice)," Proc. SPIE 9606, Instruments, Methods, and Missions for Astrobiology XVII, 960614 (11 September 2015); doi: 10.1117/12.2214475

SPIE Event: SPIE Optical Engineering + Applications, 2015, San Diego, California, United States 


\section{Pluto images clinch the existence of water, methane, and life on this typical dark matter planet (Withdrawal Notice)}

Proc. SPIE 9606, 960614 (2015)

Online Publication Date: 11 September 2015

Withdrawn from Publication: 15 October 2015

Conference Date: 9-11 August 2015

Conference Location: San Diego, California, United States

Conference Title: Instruments, Methods, and Missions for Astrobiology XVII

Conference Chairs: Richard B. Hoover, Gilbert V. Levin, Alexei Yu. Rozanov, Nalin C. Wickramasinghe 